\title{
Integrated bioinformatics analysis to identify key genes related to the prognosis of esophageal squamous cell carcinoma
}

\author{
Ying Yang, Zhiwei Sun, Youwu Shi, Jing Sun, Xiaodong Zhang \\ The VIP-II Gastrointestinal Cancer Division of Medical Department, Key Laboratory of Carcinogenesis \& Translational Research (Ministry of \\ Education), Peking University Cancer Hospital \& Institute, Beijing, China \\ Contributions: (I) Conception and design: Y Yang, X Zhang; (II) Administrative support: X Zhang; (III) Provision of study materials or patients: Y \\ Yang, Z Sun, Y Shi, J Sun; (IV) Collection and assembly of data: Y Yang, Y Shi, J Sun; (V) Data analysis and interpretation: Y Yang, Z Sun; (VI) \\ Manuscript writing: All authors; (VII) Final approval of manuscript: All authors. \\ Correspondence to: Xiaodong Zhang. The VIP-II Gastrointestinal Cancer Division of Medical Department, Key Laboratory of Carcinogenesis \& \\ Translational Research (Ministry of Education), Peking University Cancer Hospital and Institute, Beijing, China. Email: zhangxd0829@163.com.
}

Background: Esophageal squamous cell carcinoma (ESCC) is a serious threat to human health and life. The National Center for Biotechnology Information Gene Expression Omnibus (NCBI-GEO) database provides valuable information on genes related to the pathogenesis and prognosis of ESCC, which helps us to make in-depth understanding about the disease and improve its prognosis.

Methods: Four microarray profiles [GSE77861 (African Americans), GSE26886 (Germans), GSE17351 (Americans), and GSE45670 (Chinese)] from the NCBI-GEO including 49 ESCC tissues and 41 corresponding normal tissues were collected. Integrated bioinformatics methods, including proteinprotein interaction (PPI) network analysis, Gene Ontology (GO) and Kyoto Encyclopedia of Genes and Genomes (KEGG) pathway enrichment analyses, and Kaplan-Meier plotter were applied to determine the differentially expressed genes (DEGs) in ESCC together with their core functions and relationship with survival.

Results: A total of 220 upregulated and 112 downregulated genes were identified as DEGs in ESCC, of which, 40 upregulated genes were core function genes. The DEGs were mostly involved in DNA replication and cell cycle pathways. Survival analysis and Bonferroni adjustment showed kinesin family member 18A (KIF18A) and TTK protein kinase (TTK) to be related to prognosis in ESCC.

Conclusions: The findings of the present study verified the previously proposed association between TTK and patient survival in ESCC, and identified KIF18A as ESCC prognosis-related gene markers for the first time. The underlying mechanism needs to be further investigated using larger sample size studies and biological experiments in future.

Keywords: Bioinformatics analysis; prognosis; esophageal squamous cell carcinoma (ESCC)

Submitted Nov 07, 2020. Accepted for publication Feb 26, 2021.

doi: $10.21037 /$ tcr-20-3220

View this article at: http://dx.doi.org/10.21037/tcr-20-3220

\section{Introduction}

Esophageal cancer presents a serious threat to human health and life. In 2018, there were an estimated 572,034 new cases of esophageal cancer and 508,585 deaths, which saw this disease rank $7^{\text {th }}$ for incidence and $6^{\text {th }}$ for mortality out of all malignant tumors (1). In East Asia, esophageal squamous cell carcinoma (ESCC) is the major pathological type of esophageal cancer (2). Despite advancements in surgery, radiotherapy techniques, and systematic treatments, the prognosis of ESCC is still poor, with the 5-year survival rate standing at around $15 \%$ (3). Together with primary prevention, the promotion of early cancer screening programs, and the development of innovative therapy 
strategies, a comprehensive understanding of the genetic changes of ESCC would aid in improving the prognostic outlook for patients. Acquiring in-depth knowledge of the pathogenesis, progression, and prognosis of ESCC would help to deepen our perceptions of the disease, stratify patients by prognosis, and provide the foundation for precision medicine and targeted therapies. However, previous reports on ESCC genetic susceptibility genes have been inconclusive (4-8). More investigations on genetic alterations are needed to improve our understanding of ESCC and explore its underlying mechanisms; specifically, analysis of differentially expressed genes (DEGs) is an effective approach to identifying genes of interest in the disease.

Gene chip is a reliable and effective technique to quickly identify DEGs (9). DEGs identified using this technique can be deposited in public databanks for future reference. Access to such open data and integrated information can provide researchers with worthwhile hints for novel study ideas.

To identify genetic factors relating to the pathogenesis and prognosis of ESCC, and to explore the potential mechanisms, we conducted an integrated bioinformatics analysis based on the National Center for Biotechnology Information Gene Expression Omnibus (NCBI-GEO) microarray database. Unlike the previous studies $(4,5)$, we selected microarray datasets based on the same gene chip to reduce heterogeneity generated from experimental tests. Also, we selected gene profiles with no restrictions on patients' race, which might make our conclusion more generalizable. We present the following article in accordance with the MDAR checklist (available at http:// dx.doi.org/10.21037/tcr-20-3220).

\section{Methods}

\section{Microarray profiles}

Six gene expression profiles were collected from that NCBIGEO microarray database: GSE77861 (African Americans), GSE26886 (Germans), GSE17351 (Americans), and GSE100942, GSE45670, and GSE33810 (Chinese). Each of the 6 microarray profiles contained both ESCC and normal esophageal epitheliums tissues, and all expression data were built on GPL570 platform (HG-U133_Plus_2; Affymetrix Human Genome U133 Plus 2.0 Array).

\section{Data processing of DEGs}

GEO2R online tools were applied to identify DEGs between
ESCC and normal esophageal epithelial tissues (10). The filter criteria included $|\log \mathrm{FC}|>0$ and adjusted $\mathrm{P}<0.05$. Two Chinese datasets, GSE100942 and GSE33810, were excluded from further analysis, due to having an adjusted $\mathrm{P}$ value of $\geq 0.05$. Finally, GSE77861, GSE26886, GSE17351, and GSE45670, including 49 cancer tissues and 41 normal tissues, were included in the genetic analysis. Common DEGs among the 4 microarray datasets were determined using a Venn diagram (http://bioinformatics.psb.ugent.be/ webtools/Venn/). DEGs were considered to be upregulated if $\log \mathrm{FC}>0$, and downregulated if $\log \mathrm{FC}<0$.

\section{PPI network analysis}

The protein-protein interaction (PPI) network of the identified DEGs was constructed by estimating the probable interactions between the DEGs using the online instrument Search Tool for the Retrieval of Interacting Genes (STRING) in Cytoscape under the conditions of maximum number of interactions 0 and confidence score $\geq 0.4(11,12)$. The Cytoscape Molecular Complex Detection app was applied to build modules of the PPI network, with the degree cutoff set at 2, maximum depth at 100, k-core at 2 , and node score cutoff at 0.2 .

\section{Gene Ontology (GO) and patbway enrichment analysis}

Based on high-throughput transcriptomic or genomic data, we used the bioinformatics tool Database for Annotation, Visualization and Integrated Discovery (DAVID) to conduct a GO analysis of hub DEGs to analyze their genetic features, including molecular functions (MFs), cellular components (CCs), and biological processes (BPs) (Bonferroni-corrected $\mathrm{P}<0.05 /$ counts) $(13,14)$. DAVID was also employed to visualize Kyoto Encyclopedia of Genes and Genomes (KEGG) biological pathways $(\mathrm{P}<0.05)$ $(15,16)$.

\section{Survival analysis}

Associations between the identified hub genes and ESCC survival in public genome databases, including the European Genome-phenome Archive (EGA), The Cancer Genome Atlas (TCGA), and GEO (Affymetrix microarrays only), were evaluated using the online Kaplan-Meier plotter (17). The results were presented as hazard ratios (HRs) with $95 \%$ confidence intervals (CIs) and as the log-rank P value. Because of the large number of genes to be tested, 

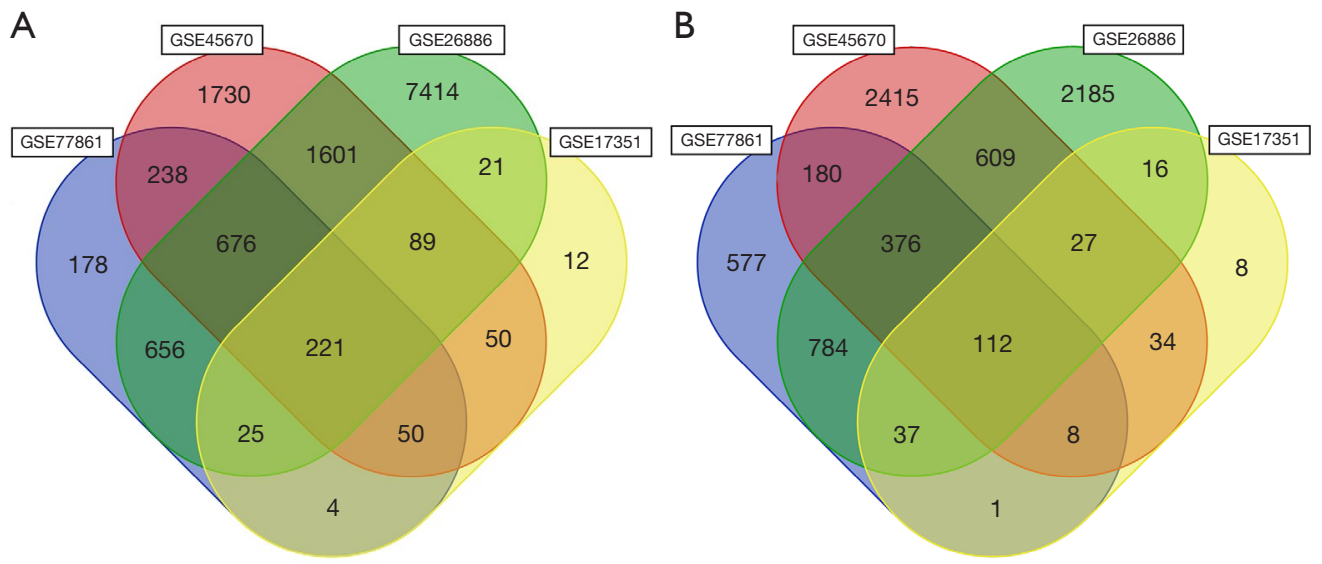

Figure 1 Common DEGs in the 4 microarray datasets via Venn diagram. Blue represents GSE77861, pink represents GSE45670, green represents GSE26886, and yellow represents GSE17351. (A) A total of 221 upregulated DEGs (included 1 unknown gene) and (B) 112 downregulated DEGs. DEGs, differentially expressed genes.

we applied both Bonferroni-corrected $\mathrm{P}<0.05 /$ the number of genes and false discovery rate (FDR) $<0.2$ to adjust for multiple comparisons.

This study was conducted in accordance with the Declaration of Helsinki (as revised in 2013). All data used in our study are available in public databases, including the NCBI-GEO microarray database, EGA, and TCGA. Individual consent for this analysis was waived.

\section{Results}

A total of 2,720 upregulated genes and 2,725 downregulated genes were obtained from GSE77861 (African Americans); 15,133 upregulated genes and 6,263 downregulated genes were obtained from GSE26886 (Germans); 567 upregulated genes and 301 downregulated genes were obtained from GSE17351 (Americans); and 6,428 upregulated genes and 5,572 downregulated genes were obtained from GSE45670 (Chinese). The DEGs identified from 49 cancer tissues and 41 normal matching tissues in the above 4 microarray profiles were compared. After the exclusion of unknown genes, 332 common DEGs, including 220 upregulated genes and 112 downregulated genes, were identified (Figure 1 and Table 1).

STRING was used to identify the nodes with the most connections as core function genes (Figure S1). As shown in Figure 2, there were 40 hub genes, all of which were upregulated.

The 40 core DEGs were subjected to GO and KEGG pathway enrichment analyses. In terms of BPs, the DEGs were significantly enriched in cell division, mitotic nuclear division, DNA replication and sister chromatid cohesion, chromosome segregation, DNA replication initiation, and G1/S transition of mitotic cell cycle. In regard to CCs, the DEGs were significantly enriched in the nucleoplasm, nucleus, chromosome, centromeric region, midbody, and spindle midzone. In terms of MFs, the DEGs were mostly involved in ATP binding, protein binding, ATPdependent microtubule motor activity, plus-end-directed, and chromatin binding (Table 2).

The KEGG pathway enrichment analysis revealed that the DEGs were mostly involved in the DNA replication and cell cycle pathways. The implicated genes included replication factor $\mathrm{C}$ subunit 4 (RFC4), minichromosome maintenance complex component 2 (MCM2), MCM5, MCM6, cell division cycle 6 (CDC6), and TTK protein kinase (TTK) (Figure 3A,B; Table 3).

Finally, we conducted a survival analysis of 39 identified core DEGs, as PICALM interacting mitotic regulator $(F A M 64 A)$ was excluded due to a lack of survival data. With $\mathrm{P}<0.05$ indicating statistical significance, 15 genes were found to be statistically significantly related to survival and are shown in Figure 4. The survival curves of the other 24 genes are presented in Figure $\mathrm{S} 2$.

The GO analysis of the 15 significant genes revealed that the most enriched BP, CC, and MF were cell division, nucleoplasm, and ATP binding, respectively (Table 4). KEGG pathway enrichment analysis again showed enrichment mainly in the cell cycle pathway (Figure $3 B$ and Table 5). 
Table 1 Common differentially expressed genes (DEGs) identified from 4 microarray datasets of esophageal squamous cell carcinoma tissues compared with normal esophageal tissues $(n=332)$

\begin{tabular}{ll}
\hline DEGs & Gene name \\
\hline Upregulated & KRT17 IPO9 CDC25B NUDT1 FOXM1 PLXNA1 DDX18 KIAA0930 KIF4A MEX3D MCM5 TMEM132A MIR1292 CD276 \\
genes (n=220) & MIR1182 PAPD7 CDCA2 PTRH2 OTULIN RPN2 APOC1 GPNMB FZD2 KIF18B ENAH ATP2C1 TMPO MBD4 CSE1L \\
& CENPN RFC4 MIR664B TMEM9 UBE2S NIFK OCIAD2 NTMT1 TRAF4 WDR5 PHF20L1 DESI2 E2F6 FZD6 CUX1 \\
& CKAP5 HDGF ATP1B3 DHX36 NUDCD1 PAK2 ATP2B1 FAM64A PFDN2 ITPR3 U2SURP DDX11 IMP4 RIOK1 DSCC1 \\
& KNTC1 LRRC8D SAC3D1 AURKB GANAB GTSE1 NUF2 CDCA5 TEAD4 YWHAG IGF2BP2 WDR53 PSMB4 TMEM97 \\
& LAMC2 IMPDH1 ASXL1 IGF2R COLGALT1 BEND3 LPCAT1 TCOF1 DNAH14 HMGB3 HOMER3 MRGBP TSEN15 \\
& MYBL2 CENPO CKS1B BLM DIEXF CLCN7 PDCD2L NAPEPLD GABPB1 TFRC PSME4 PARP1 SERPINH1 CDCA3 \\
& SIX4 FLAD1 TCF3 DTL MEST GMNN CASC7 TTK SLC52A2 MAPKAPK2 FOXK2 HSPD1 RSRC1 COL7A1 KIF14 \\
& MCM10 ELF4 NELL2 CHPF2 SPAG5 AGRN CLPTM1L EML4 MCM2 TGS1 CBX3 MARCKSL1 MTERF3 CENPI MIR1178 \\
& ARFGAP1 BID NAXE ABL2 ITGB4 ABCC4 RIF1 HOXA10 RUVBL1 ADO ACVR1C SCARB1 TRIP13 FNDC3B HEATR1 \\
& DHX9 DDX28 UBE2C TOP2A HELLS USB1 KIF18A CACYBP MCM6 DERL1 CTHRC1 UHRF1 SGO2 ZAK CENPF \\
& NDUFB9 BYSL TPX2 ANP32E PUF60 DNMT3B CDC6 AURKA CHAF1A RAD1 DDX39A ASAP1 RUNX1 ITGA6 PRKDC \\
& NOL11 C1Orf131 UBE2T PTDSS1 ECT2 CDH3 ARPC1B RAD51AP1 MIR7112 MMP10 EPHB4 HMCES PLAU UTP4 \\
& EIF3B FLVCR1 PCYT1A SLC25A32 YEATS2 SNRNP200 DHX33 APMAP C4Orf48 ZNF281 ISG20L2 TNFAIP8L1 ADRM1 \\
& TMEM184B MED30 NCAPH ATAD2 DNMT1 CAD RPA1 UBAP2L TMEM138 REL MLEC RTKN KIAA1804 ORC6 GMPS \\
& DBR1 WDR66 CTSC
\end{tabular}

Downregulated C4orf3 CPEB3 ALAD PAQR8 UFL1 TFAP2B AHNAK RSU1 CHMP2B CUL4B KALRN CALCOCO2 FBXL5 RBP7 genes $(\mathrm{n}=112) \quad$ EPB41L3 PIAS1 FIG4 CCSER2 CITED2 ABHD5 SLC6A1 TRIOBP NCOA1 FBXL3 NOL12 BBIP1 PLA2G12A KANK1 PTN MDFIC EPB41L4A HSPB8 RAP1A SPAG16 LNPEP PGRMC2 FYCO1 ANKRD35 DDAH1 PRMT2 HACE1 GID4 SLC30A4 TLR3 STX17 NUCB2 UBL3 SECISBP2L KCTD6 SHROOM3 ANKHD1-EIF4EBP3 COBL RAB11A RRAGD CNPPD1 PHYHD1 AR PINK1 C15orf52 ALDH3A2 PDZRN3 BCAP29 VIT SH3BGRL2 KCNAB1 SAMD5 SSBP2 CTTNBP2 SASH1 PHC1 GNE ARL6IP5 MYZAP DCAF10 LOC100132167 FAM219B AZI2 USO1 HSDL2 PPP1R7 KAT2B SLC30A9 SMDT1 LMBRD1 SCIN EMP1 EDN3 AK9 MTERF4 HPGD CPPED1 GAB2 FAM13C DOCK9 MGLL RORA ATP5O TRIM13 ENSA GNAQ SNX3 EIF4E3 CAPN5 FAM189A2 PCCA DEPTOR DENND4C CAB39L ABLIM3 GGTA1P CAST GAB1

DEGs, differentially expressed genes.

For multiple comparisons, we applied a Bonferroni correction of $0.05 / 39=0.001282$ as Bonferroni-adjusted statistical significance. We also applied FDR $<0.20$ as a second reference. Eventually, 2 genes were identified as being significantly related to survival: kinesin family member 18A (KIF18A) (HR =0.24, 95\% CI: 0.10-0.59, logrank $\mathrm{P}=0.00072, \mathrm{FDR}=0.10)$ and TTK $(\mathrm{HR}=0.20,95 \%$ CI: $0.07-0.55$, log-rank $\mathrm{P}=0.00057$, FDR =0.10). Patients with a high expression of these genes had a better prognosis than those with a low expression.

\section{Discussion}

By performing a gene enrichment bioinformatics analysis of ESCC which did not factor in race, we identified 332 common DEGs from 4 microarray datasets (GSE77861, GSE26886, GSE17351, and GSE45670). Through module analysis, we identified 40 upregulated genes as core function genes, and the most involved pathways were the DNA replication and cell cycle pathways, which supported our previous knowledge of carcinogenesis. In the prognostic analysis, we identified 15 genes associated with survival. For these survival-related genes, the most enriched BP, CC, and MF were cell division, nucleoplasm, and ATP binding, respectively, and the most enriched pathway was the cell cycle pathway. Furthermore, for multiple comparisons, we applied the Bonferroni correction and FDR, and identified 2 genes, KIF18A (HR =0.24, 95\% CI: 0.10-0.59, log-rank $\mathrm{P}=0.00072, \mathrm{FDR}=0.10)$ and TTK $(\mathrm{HR}=0.20,95 \% \mathrm{CI}$ : $0.07-0.55$, log-rank $\mathrm{P}=0.00057$, FDR $=0.10$ ), as prognosisrelated genes.

Although our findings present associations between KIF18A/TTK and ESCC development and prognosis, the exact mechanisms still are unclear, especially as research on these aspects of ESCC is lacking. A number of in vitro studies have explored the functional role of $T T K$ in cancer, and have discovered the major pathways involved to be the $T T K-A K T$-mechanistic target of rapamycin kinase $(m T O R)$ pathway in hepatocellular carcinoma and TTK/ $A K T$ interaction with $B-R a f /$ mitogen-activated protein 


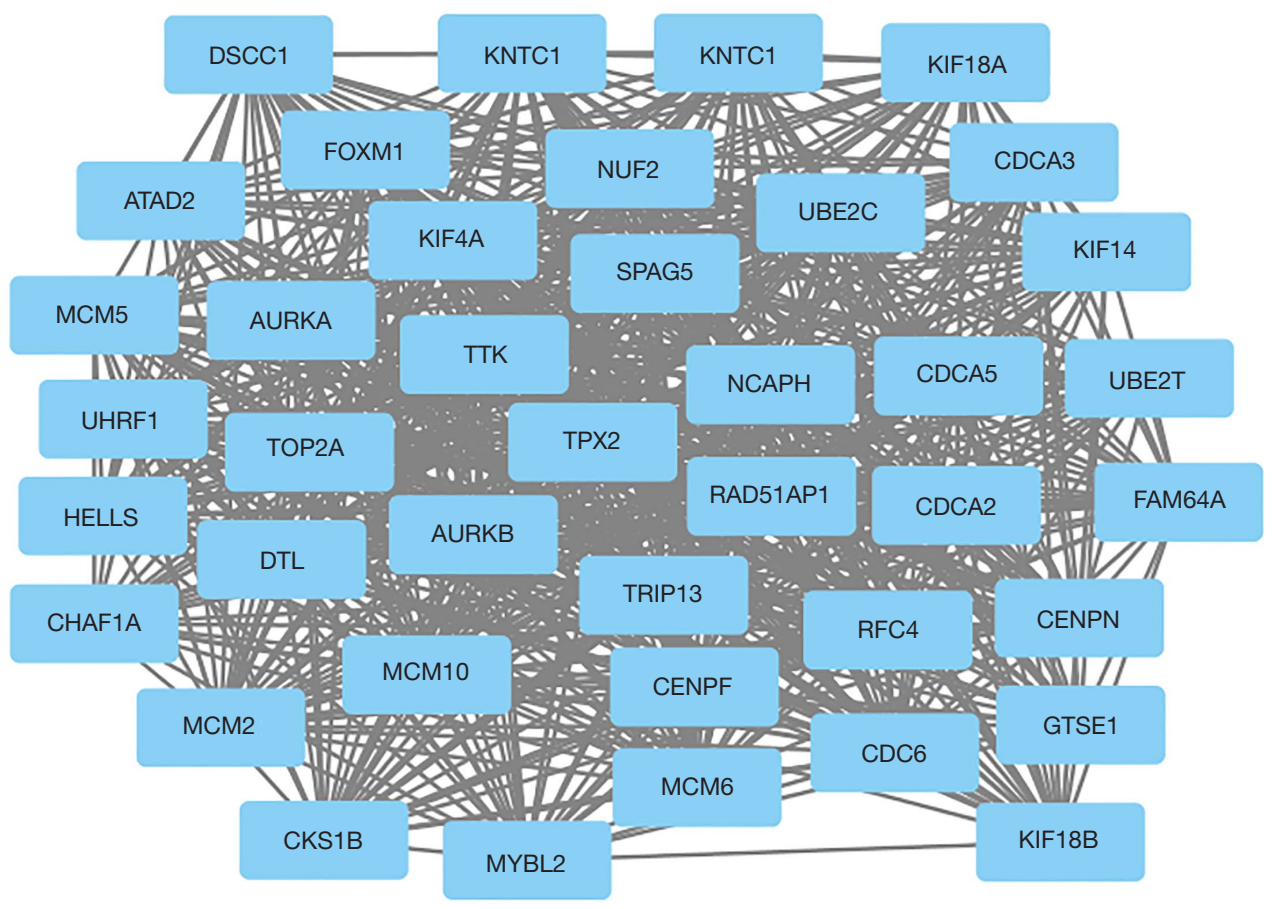

Figure 2 The 40 most connected nodes in module analysis via Cytoscape. Each blue square represents a gene, and the connected lines represent interactions between genes.

kinase 1 (MAPK1) signals in melanoma (18,19). KIF18A, KIF18B, and KIF19 are kinesin- 8 family members. KIF18A together with dyneins, that are as the key component of canonical plus-end stepping in the mitotic spindle and the depolymerization of microtubules, is primarily involved in mitotic chromosome alignment, maintenance of chromosomal stability, and cell division. It also acts as the major driving force for cell migration (20-26). KIF18A dysregulation may give rise to genomic instability (27), eventually resulting in neoplasm development and metastatic progression (28).

Earlier studies have shown that the overexpression of human KIF18A is related to a poor prognosis of colorectal cancer (20), invasive breast cancer (29), and primary hepatocellular carcinoma (30). However, studies on KIF18A in ESCC are scarce. To the best of our knowledge, the present study is the first to reveal the relationship of KIF18A with the prognosis of ESCC. Taken together, previously published findings $(20,29,30)$ and the results of the present study imply that KIF18A is a possible prognostic predictor; however, the molecular mechanisms need further elucidation.

In terms of treatment, the mechanism and molecules relevant to mitosis are well-established targets for microtubule-binding chemotherapeutic agents, such as taxol and vinca alkaloids (31), which is consistent with the fact that regimens containing paclitaxel are the preferred chemotherapeutic treatment for ESCC in Chinese clinical practice (32). Additionally, mitotic kinesins have also been considered as potentially valuable targets in the development of anticancer drugs (33).

TTK is a cancer/testis antigen (CTA) and an HLAA2402-restricted epitope peptide (34). As well as in the testes, early-evolving embryos, the thymus, and placentas, the expression of CTA can be detected in various malignant tumors, and it is rarely detected in benign tissues $(35,36)$. TTK participates in cell proliferation and migration through the phosphorylation of multiple amino acids, such as serine, threonine, and tyrosine hydroxyamino acids, and functions to maintain genomic integrity through regulating the spindle assembly checkpoint (35,37-39).

Previous studies have revealed the associations between TTK overexpression and the growth of pancreatic ductal adenocarcinoma (40), hepatocellular carcinoma (18), breast cancer (41), and melanoma (19). A previously published study found that TTK was a key component in ESCC 


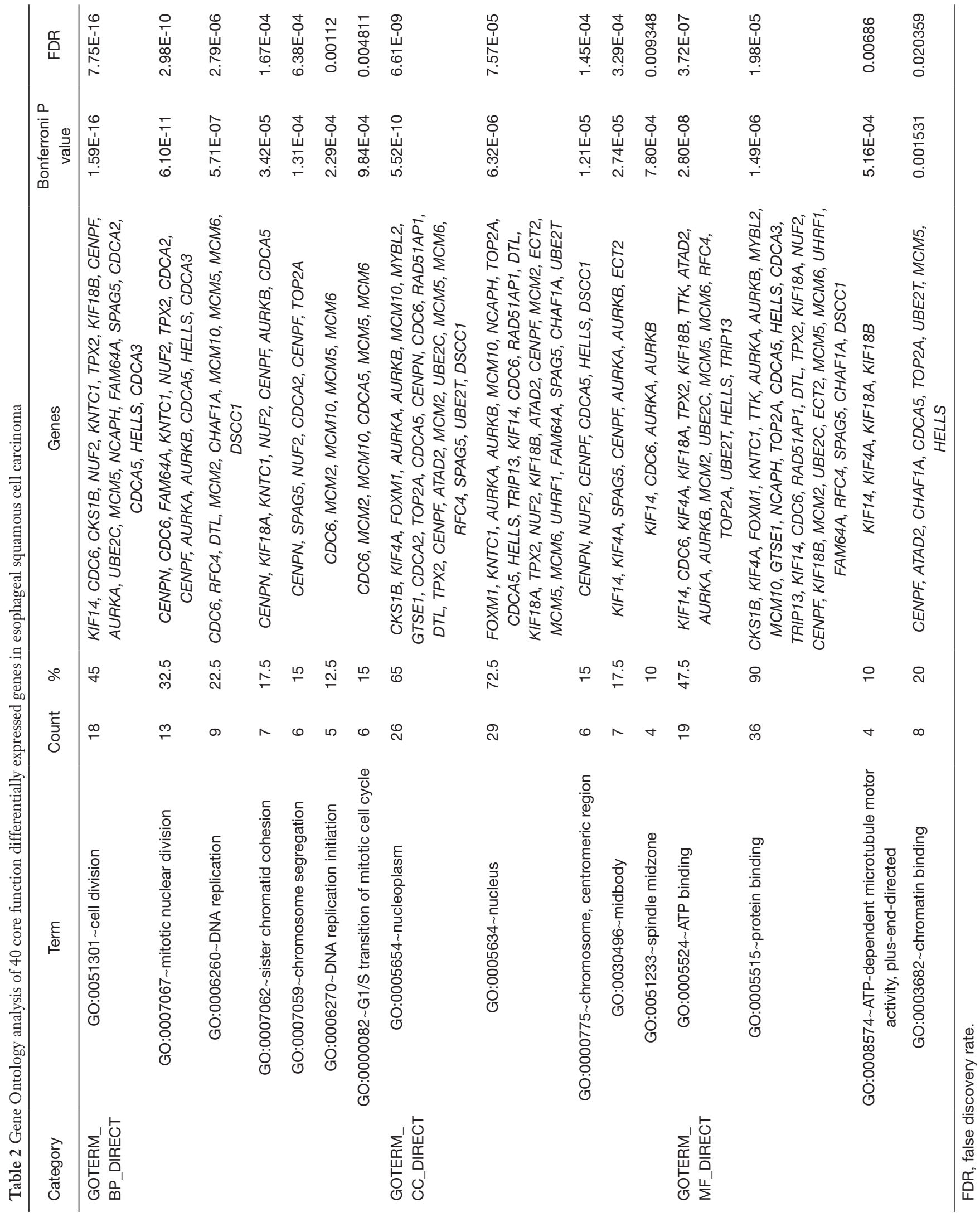


A DNA REPLICATION
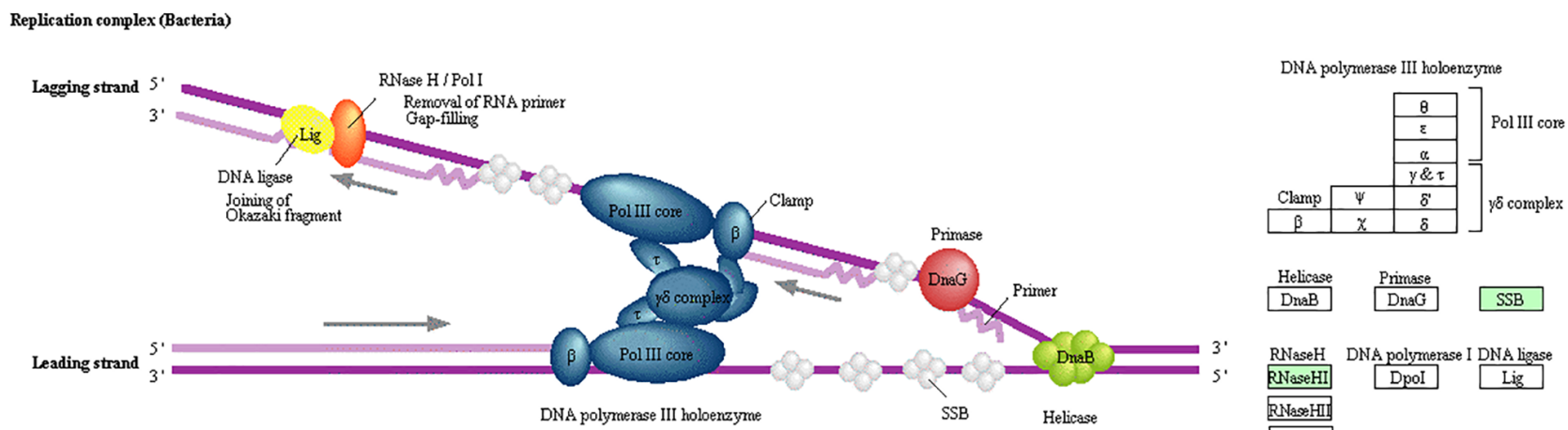

RNaseH DNA polymerase I DNA ligase

NaseeH Dpol Lig

DNA polymerase III holoenzyme

SSB

RNatin

Replication complex (Anchaea)

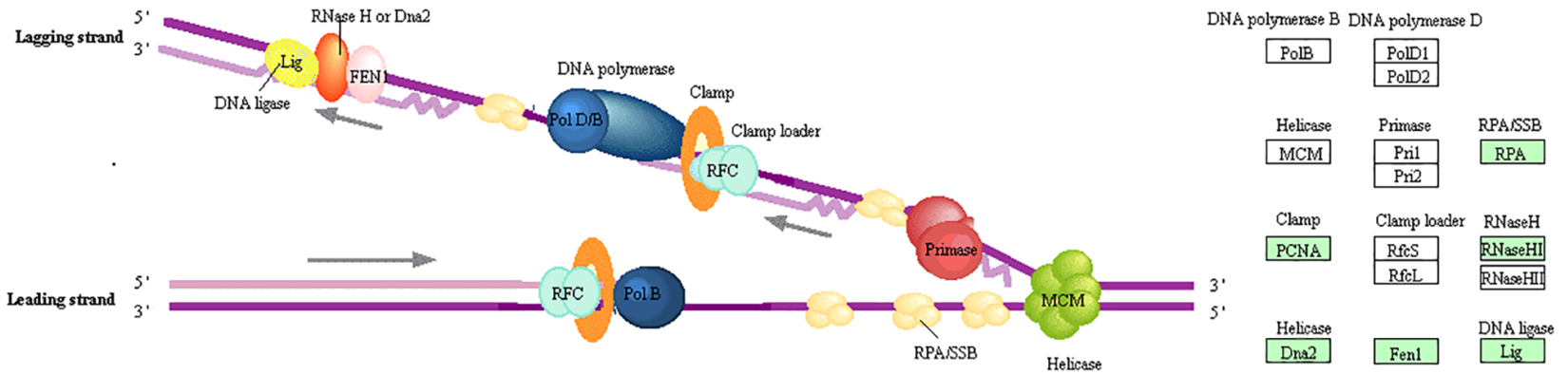

Replication complex (Eukaryotes)

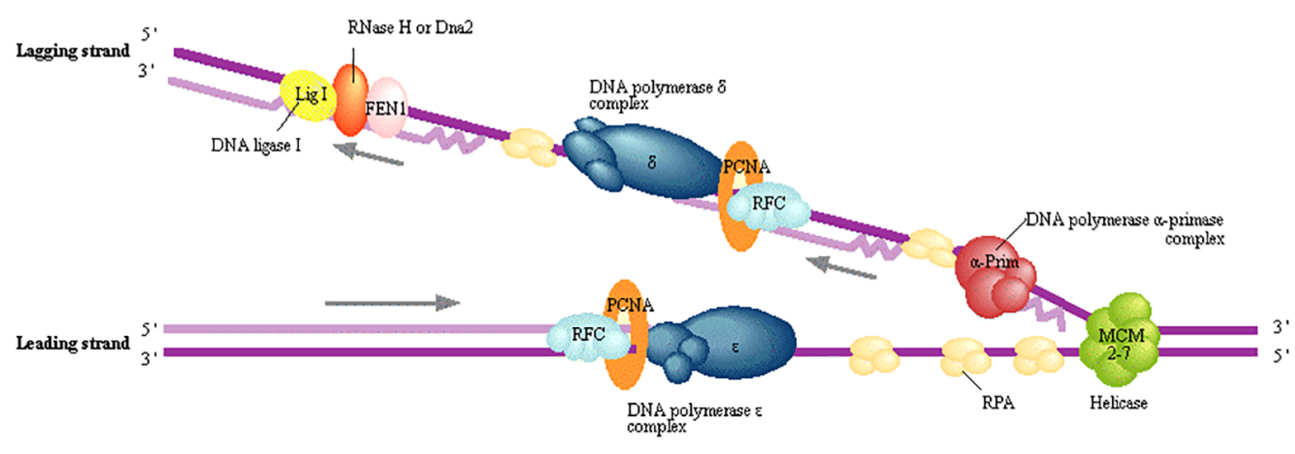

DNA polymerase c-primase complex \begin{tabular}{|l|l|l|l|}
\hline$\alpha 1$ & $\alpha 2$ & Pril & Pri2 \\
\hline
\end{tabular}

DNA polymerase $\delta$ complex \begin{tabular}{|l|l|l|l|}
\hline$\delta 1$ & $\delta 2$ & $\delta 3$ & $\delta 4$ \\
\hline
\end{tabular}

DNA polymerase $\varepsilon$ complex \begin{tabular}{|c|c|c|c|}
\hline$\varepsilon 1$ & $\varepsilon 2$ & $\varepsilon 3$ & $\varepsilon 4$ \\
\hline
\end{tabular}

MCM complex (helicase)
\begin{tabular}{|l|l|}
\hline Mcm2 & Mcm3 \\
\hline Mcm4 & Mcm5 \\
\hline Mcm6 & Mcm? \\
\hline
\end{tabular}$\quad$\begin{tabular}{|c|}
\hline RFA1 \\
\hline RFA2/4 \\
\hline RPA3 \\
\hline
\end{tabular}

Clamp Clamp loader \begin{tabular}{|l|l|l|l|}
\hline PCNA & RFC1 & RFC2/4 & RFC3/5 \\
\hline
\end{tabular} RNaseHI $\frac{\text { RNaseHII }}{\text { RNaseHI }}$ \begin{tabular}{lll} 
Helicase & \\
\hline Dna2 & DNA ligase \\
\hline
\end{tabular}

$0303012118 / 17$
(c) Kanehisa Laboratories 


\section{B}

CELL CYCLE

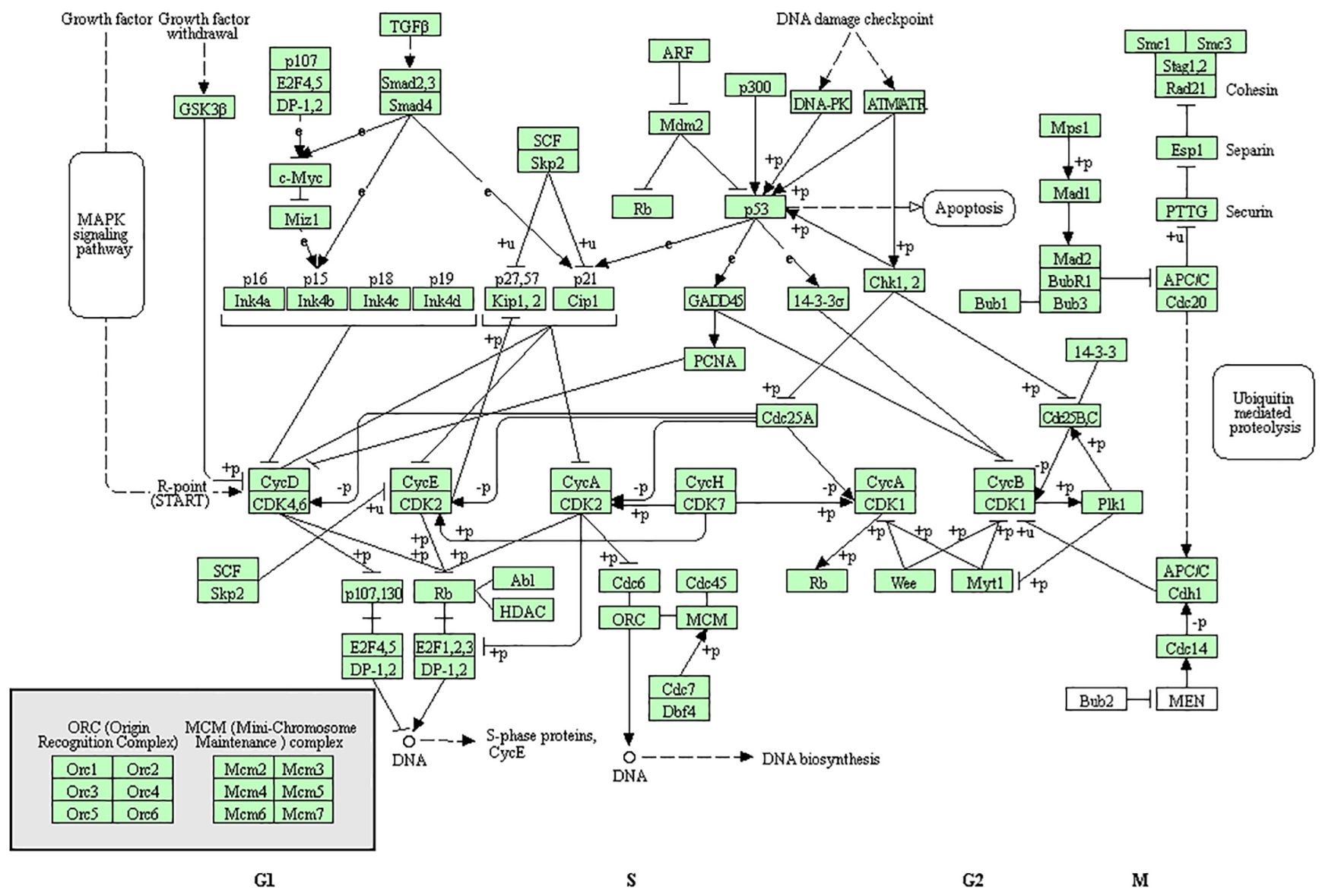

$0411011 / 15 / 18$

(c) Kanehisa Laboratories

Figure 3 The Kyoto Encyclopedia of Genes and Genomes (KEGG) pathway enrichment analysis of the 40 core differentially expressed genes in esophageal squamous cell carcinoma. (A) KEGG pathway enrichment of hsa03030: DNA replication. Involved genes were replication factor C subunit 4 (RFC4), minichromosome maintenance complex component 2 (MCM2), MCM5, MCM6. (B) KEGG pathway enrichment of hsa04110: cell cycle. Involved genes were cell division cycle 6 (CDC6), TTK protein kinase (TTK), MCM2, MCM5, and MCM6.

Table 3 Kyoto Encyclopedia of Gene and Genome pathway analysis of 40 core differentially expressed genes in esophageal squamous cell carcinoma

\begin{tabular}{lccccc}
\hline Term & Count & $\%$ & P-value & Genes \\
\hline hsa03030: DNA replication & 4 & 10 & $2.11 \mathrm{E}-05$ & RFC4, MCM2 MCM5, MCM6 \\
hsa04110: Cell cycle & 5 & 12.5 & $3.01 \mathrm{E}-05$ & CDC6, TTK, MCM2MCM5, MCM6 & 0.013148 \\
\hline
\end{tabular}

FDR, false discovery rate.

progression and predicted a poor prognosis, although the molecular mechanisms were unclear (4).

TTK inhibitors suppress the activity of monopolar spindle 1 kinase, specifically deactivate the spindle assembly checkpoint, which results in chromosome separation dysfunction, heteroploidy, and ultimately, cell death. Thus, TTK inhibition has arisen as a promising therapeutic strategy for triple-negative breast cancer and malignant 
A

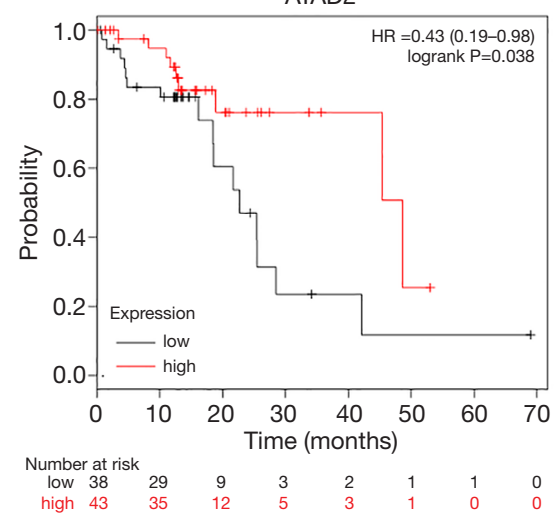

D

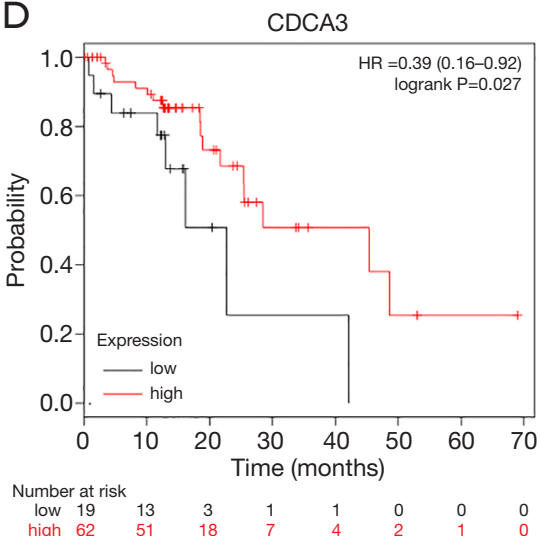

G

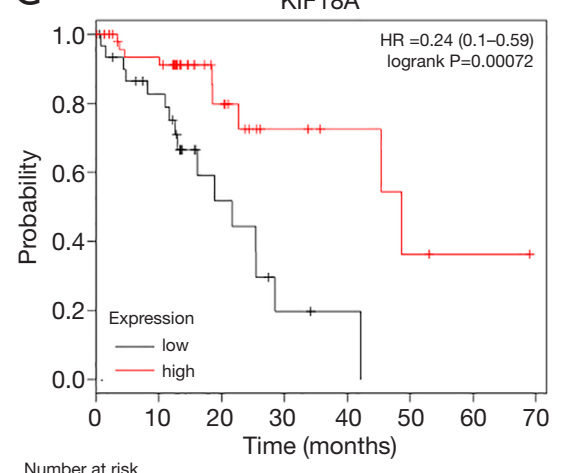

B

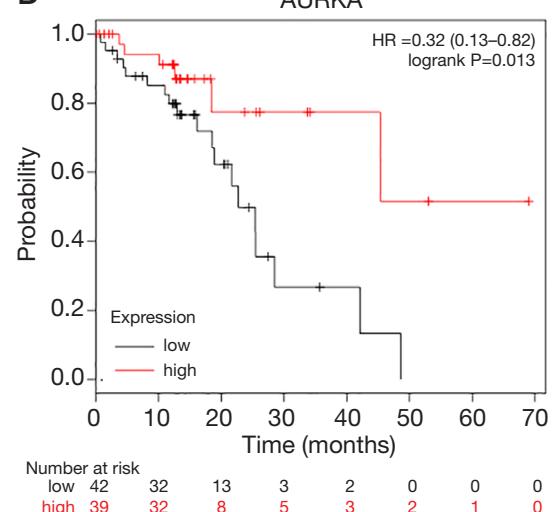

E

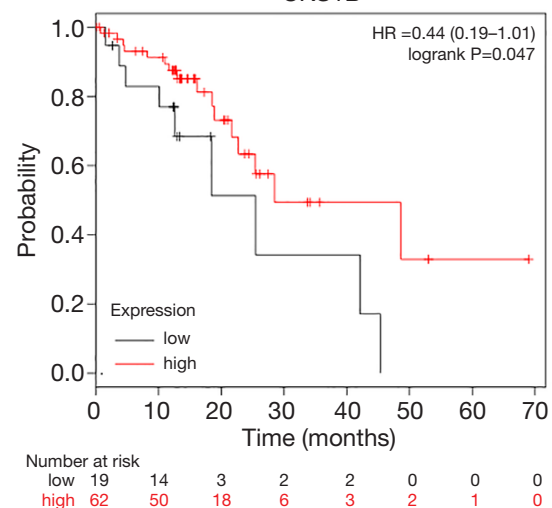

$\mathrm{H}$

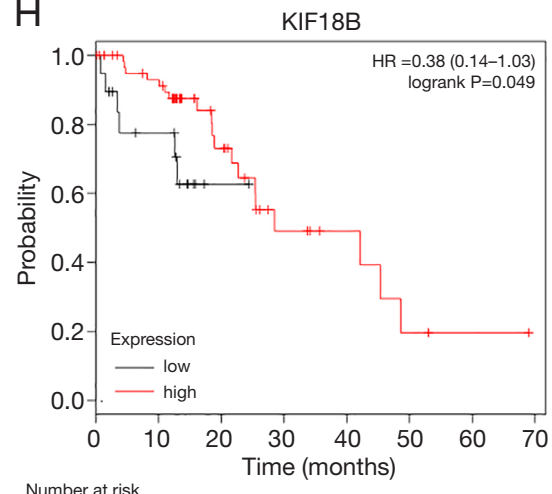

C

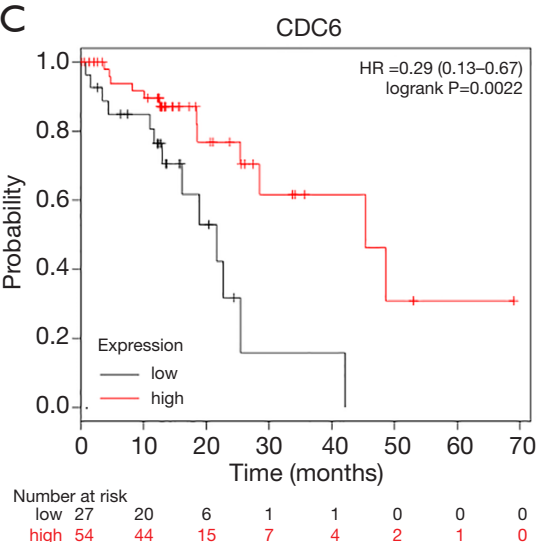

$\mathrm{F}$

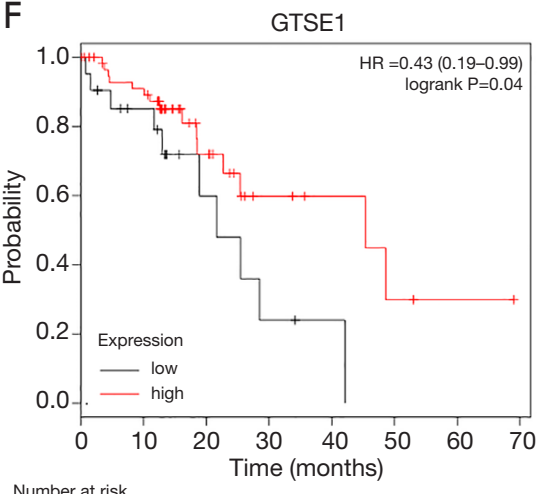

$\begin{array}{cccccccc}\begin{array}{c}\text { Number at risk } \\ \text { low } 21\end{array} & 14 & 5 & 2 & 1 & 0 & 0 & 0 \\ \text { high } 60 & 50 & 16 & 6 & 4 & 2 & 1 & 0\end{array}$

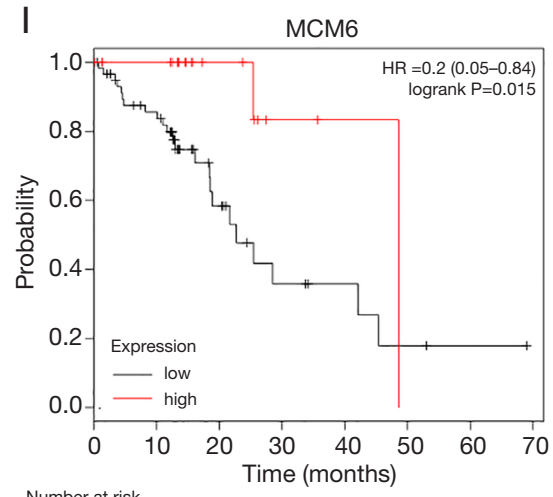

$\begin{array}{cccccccc}\text { Number at risk } & & & & & & \\ \text { low } 60 & 45 & 14 & 6 & 4 & 2 & 1 & 0 \\ \text { high } 21 & 19 & 7 & 2 & 1 & 0 & 0 & 0\end{array}$ 

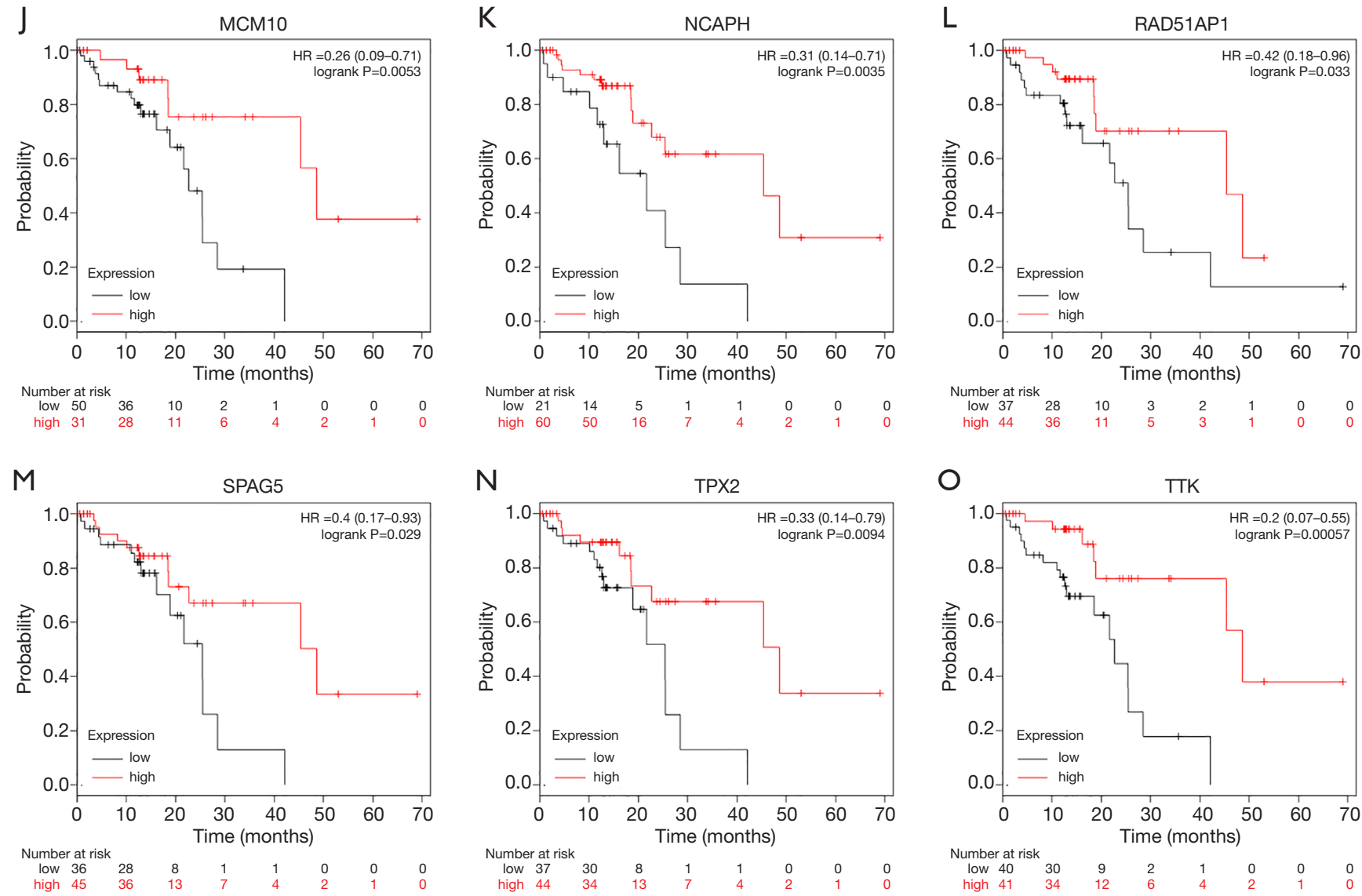

Figure 4 Kaplan-Meier plots of the 15 genes with statistical significance $(\mathrm{P}<0.05)$. Gene name is shown at the top of each figure part. Black line represents low expression of the identified gene, and red line represents high expression of the identified gene. Corresponding life table, hazard ratio, and log-rank $\mathrm{P}$ value are shown in each figure part.

Table 4 Gene Ontology analysis of 15 differentially expressed genes related to esophageal squamous cell carcinoma survival

\begin{tabular}{|c|c|c|c|c|c|}
\hline Category & Count & $\%$ & Genes & $\begin{array}{l}\text { Bonferroni } \mathrm{P} \\
\text { value }\end{array}$ & FDR \\
\hline $\begin{array}{l}\text { GOTERM_BP_ GO:0051301 cell division } \\
\text { DIRECT }\end{array}$ & 8 & 53.33333 & $\begin{array}{c}\text { CKS1B, CDC6, NCAPH, SPAG5, TPX2, } \\
\text { KIF18B, AURKA, CDCA3 }\end{array}$ & 5.17E-07 & 5.47E-06 \\
\hline $\begin{array}{l}\text { GOTERM_CC_GO:0005654 nucleoplasm } \\
\text { DIRECT }\end{array}$ & 10 & 66.66667 & $\begin{array}{c}\text { CKS1B, CDC6, RAD51AP1, SPAG5, TPX2, } \\
\text { ATAD2, AURKA, MCM10, GTSE1, MCM6 }\end{array}$ & 0.002063 & 0.041061 \\
\hline
\end{tabular}

FDR, false discovery rate.

mesothelioma (37,42-46). Furthermore, the testis is an immunoprivileged organ, and CTAs are ideal candidates as tumor-related antigens (47). TTK has emerged as a valuable target for tumor immunotherapy, including cancer vaccines, chimeric antigen receptor $\mathrm{T}$-cell immunotherapy, and immune checkpoint inhibitors, due to its cancerspecific expression and strong in vivo immunogenicity, together with its critical roles in cell proliferation and 
Table 5 Kyoto Encyclopedia of Genes and Genomes pathway analysis of 15 differentially expressed genes related to esophageal squamous cell carcinoma

\begin{tabular}{lccccc}
\hline Term & Count & $\%$ & P value & Genes & FDR \\
\hline hsa04110: cell cycle & 3 & 20 & 0.003111 & CDC6, TTK, MCM6 & 1.480007 \\
\hline
\end{tabular}

FDR, false discovery rate.

migration, and spindle assembly checkpoint regulation $(42,47,48)$. In several pharmaceutical clinical trials on ESCC, TTK expression was reported as both a marker for the treatment response and a potential cancer vaccine target $(49,50)$.

Our study has several limitations. First, a previous published study conducted using the laiurger microarray dataset (GSE38129 and GSE20347) recognized that the TPX2 microtubule nucleation factor, cyclin dependent kinase 1 , and centrosomal protein 55 were associated with relapse-free survival (51). Instead, we selected another microarray dataset built on GPL570 platform, including diverse races, and chose overall survival as our study endpoint rather than relapse-free survival, which resulted in our conclusion being inconsistent with those of earlier studies. However, the discrepancy between our research and previous research revealed that the bioinformatics analysis results were unstable and emphasized the significance of molecular mechanism studies. Second, the survival data on ESCC were limited, and the small sample size reduced the reliability of the results; therefore, larger sample sizes are required in future research. Third, for both KIF18A or TTK, molecular mechanism studies were limited and none of them focused on ESCC. It should be recognized that gene enrichment analysis can only provide clues, and our findings should be further verified by biological experiments. Finally, bioinformatics analysis is a growing field. New analyses and gene expression microarray studies are in development, which might affect our conclusions. The challenge for researchers performing bioinformatics analyses is to integrate up-to-date analytical methods and datasets accordingly.

In conclusion, our analysis was the first to identify KIF18A related to ESCC prognosis and verified the previous association between TTK and ESCC survival. KIF18A is a key component of mitotic activity, and TTK is a good candidate for tumor immunotherapy. The results of the present study were in accordance with those of studies on the clinical use of paclitaxel and also shed light on directions for future anticancer immunotherapy research and development. However, our conclusions need to be further validated in studies with larger sample sizes and biological experiments.

\section{Acknowledgments}

Funding: None.

\section{Footnote}

Reporting Checklist: The authors have completed the MDAR checklist. Available at http://dx.doi.org/10.21037/tcr-203220

Conflicts of Interest: All authors have completed the ICMJE uniform disclosure form (available at http://dx.doi. org/10.21037/tcr-20-3220). The authors have no conflicts of interest to declare.

Ethical Statement: The authors are accountable for all aspects of the work in ensuring that questions related to the accuracy or integrity of any part of the work are appropriately investigated and resolved. The study was conducted in accordance with the Declaration of Helsinki (as revised in 2013). All data used in our study were available in public database, and individual consent for this analysis was waived.

Open Access Statement: This is an Open Access article distributed in accordance with the Creative Commons Attribution-NonCommercial-NoDerivs 4.0 International License (CC BY-NC-ND 4.0), which permits the noncommercial replication and distribution of the article with the strict proviso that no changes or edits are made and the original work is properly cited (including links to both the formal publication through the relevant DOI and the license). See: https://creativecommons.org/licenses/by-nc$\mathrm{nd} / 4.0 \%$. 


\section{References}

1. Bray F, Ferlay J, Soerjomataram I, et al. Global cancer statistics 2018: GLOBOCAN estimates of incidence and mortality worldwide for 36 cancers in 185 countries. CA Cancer J Clin 2018;68:394-424.

2. Abnet CC, Arnold M, Wei WQ. Epidemiology of Esophageal Squamous Cell Carcinoma. Gastroenterology 2018;154:360-73.

3. Chen $W$, Zheng R, Baade PD, et al. Cancer statistics in China, 2015. CA Cancer J Clin 2016;66:115-32.

4. He W, Chen L, Yuan K, et al. Gene set enrichment analysis and meta-analysis to identify six key genes regulating and controlling the prognosis of esophageal squamous cell carcinoma. J Thorac Dis 2018;10:5714-26.

5. Chen FF, Zhang SR, Peng H, et al. Integrative genomics analysis of hub genes and their relationship with prognosis and signaling pathways in esophageal squamous cell carcinoma. Mol Med Rep 2019;20:3649-60.

6. Song G, Xu J, He L, et al. Systematic profiling identifies PDLIM2 as a novel prognostic predictor for oesophageal squamous cell carcinoma (ESCC). J Cell Mol Med 2019;23:5751-61.

7. Yang Q, Lin W, Liu Z, et al. RAP80 is an independent prognosis biomarker for the outcome of patients with esophageal squamous cell carcinoma. Cell Death Dis 2018;9:146.

8. Sang M, Gu L, Liu F, et al. Prognostic Significance of MAGE-A11 in Esophageal Squamous Cell Carcinoma and Identification of Related Genes Based on DNA Microarray. Arch Med Res 2016;47:151-61.

9. Vogelstein B, Papadopoulos N, Velculescu VE, et al. Cancer genome landscapes. Science 2013;339:1546-58.

10. Davis S, Meltzer PS. GEOquery: a bridge between the Gene Expression Omnibus (GEO) and BioConductor. Bioinformatics (Oxford, England) 2007;23:1846-7.

11. Damian S, Andrea F, Stefan W, et al. STRING v10: protein-protein interaction networks, integrated over the tree of life. Nucleic Acids Res 2015;43:D447-52.

12. Shannon P, Markiel A, Ozier O, et al. Cytoscape: a software environment for integrated models of biomolecular interaction networks. Genome research 2003;13:2498-504.

13. Huang DW, Sherman BT, Lempicki RA. Systematic and integrative analysis of large gene lists using DAVID bioinformatics resources. Nat Protoc 2009;4:44-57.

14. Ashburner M, Ball CA, Blake JA, et al. Gene ontology: tool for the unification of biology. The Gene Ontology
Consortium. Nat Genet 2000;25:25-9.

15. Kanehisa M, Goto S. KEGG: kyoto encyclopedia of genes and genomes. Nucleic Acids Res 2000;28:27-30.

16. Huang da W, Sherman BT, Lempicki RA. Bioinformatics enrichment tools: paths toward the comprehensive functional analysis of large gene lists. Nucleic Acids Res 2009;37:1-13

17. Szász AM, Lánczky A, Nagy Á, et al. Cross-validation of survival associated biomarkers in gastric cancer using transcriptomic data of 1,065 patients. Oncotarget 2016;7:49322-33.

18. Liu X, Liao W, Yuan Q, et al. TTK activates Akt and promotes proliferation and migration of hepatocellular carcinoma cells. Oncotarget 2015;6:34309-20.

19. Zhang L, Shi R, He C, et al. Oncogenic B-Raf(V600E) abrogates the AKT/B-Raf/Mps1 interaction in melanoma cells. Cancer Lett 2013;337:125-32.

20. Nagahara M, Nishida N, Iwatsuki $M$, et al. Kinesin 18A expression: clinical relevance to colorectal cancer progression. Int J Cancer 2011;129:2543-52.

21. Mayr MI, Hümmer S, Bormann J, et al. The human kinesin Kif18A is a motile microtubule depolymerase essential for chromosome congression. Curr Biol 2007;17:488-98.

22. Stumpff J, von Dassow $G$, Wagenbach $M$, et al. The kinesin-8 motor Kif18A suppresses kinetochore movements to control mitotic chromosome alignment. Dev Cell 2008;14:252-62.

23. Weaver LN, Ems-McClung SC, Stout JR, et al. Kif18A uses a microtubule binding site in the tail for plus-end localization and spindle length regulation. Curr Biol 2011;21:1500-6.

24. Gardner MK, Odde DJ, Bloom K. Kinesin-8 molecular motors: putting the brakes on chromosome oscillations. Trends Cell Biol 2008;18:307-10.

25. Kim H, Fonseca C, Stumpff J. A unique kinesin-8 surface loop provides specificity for chromosome alignment. Mol Biol Cell 2014;25:3319-29.

26. Locke J, Joseph AP, Peña A, et al. Structural basis of human kinesin-8 function and inhibition. Proc Natl Acad Sci U S A 2017;114:E9539-E48.

27. Cleveland DW, Mao Y, Sullivan KF. Centromeres and kinetochores: from epigenetics to mitotic checkpoint signaling. Cell 2003;112:407-21.

28. Hartwell LH, Kastan MB. Cell cycle control and cancer. Science 1994;266:1821-8.

29. Kasahara M, Nagahara M, Nakagawa T, et al. Clinicopathological relevance of kinesin family member 
18A expression in invasive breast cancer. Oncol Lett 2016;12:1909-14.

30. Liao W, Huang G, Liao Y, et al. High KIF18A expression correlates with unfavorable prognosis in primary hepatocellular carcinoma. Oncotarget 2014;5:10271-9.

31. Jordan MA, Wilson L. Microtubules as a target for anticancer drugs. Nat Rev Cancer 2004;4:253-65.

32. Kim JY, Do YR, Park KU, et al. A multi-center phase II study of docetaxel plus cisplatin as first-line therapy in patients with metastatic squamous cell esophageal cancer. Cancer Chemother Pharmacol 2010;66:31-6.

33. Rath O, Kozielski F. Kinesins and cancer. Nat Rev Cancer 2012;12:527-39.

34. Mizukami Y, Kono K, Daigo Y, et al. Detection of novel cancer-testis antigen-specific T-cell responses in TIL, regional lymph nodes, and PBL in patients with esophageal squamous cell carcinoma. Cancer Sci 2008;99:1448-54.

35. Mills GB, Schmandt R, McGill M, et al. Expression of TTK, a novel human protein kinase, is associated with cell proliferation. J Biol Chem 1992;267:16000-6.

36. Whitehurst AW. Cause and consequence of cancer/testis antigen activation in cancer. Annu Rev Pharmacol Toxicol 2014;54:251-72.

37. Thu KL, Silvester J, Elliott MJ, et al. Disruption of the anaphase-promoting complex confers resistance to TTK inhibitors in triple-negative breast cancer. Proc Natl Acad Sci U S A 2018;115:E1570-7.

38. Tao $\mathrm{Y}$, Chai $\mathrm{D}, \mathrm{Ma} \mathrm{L}$, et al. Identification of distinct gene expression profiles between esophageal squamous cell carcinoma and adjacent normal epithelial tissues. Tohoku J Exp Med 2012;226:301-11.

39. King JL, Zhang B, Li Y, et al. TTK promotes mesenchymal signaling via multiple mechanisms in triple negative breast cancer. Oncogenesis 2018;7:69.

40. Slee RB, Grimes BR, Bansal R, et al. Selective inhibition of pancreatic ductal adenocarcinoma cell growth by the mitotic MPS1 kinase inhibitor NMS-P715. Mol Cancer Ther 2014;13:307-15.

41. Györffy B, Bottai G, Lehmann-Che J, et al. TP53 mutation-correlated genes predict the risk of tumor relapse and identify MPS1 as a potential therapeutic kinase in TP53-mutated breast cancers. Mol Oncol 2014;8:508-19.

42. Mason JM, Wei X, Fletcher GC, et al. Functional characterization of CFI-402257, a potent and selective Mps1/TTK kinase inhibitor, for the treatment of cancer. Proc Natl Acad Sci U S A 2017;114:3127-32.
43. Riggs JR, Nagy M, Elsner J, et al. The Discovery of a Dual TTK Protein Kinase/CDC2-Like Kinase (CLK2) Inhibitor for the Treatment of Triple Negative Breast Cancer Initiated from a Phenotypic Screen. J Med Chem 2017;60:8989-9002.

44. Maia AR, de Man J, Boon U, et al. Inhibition of the spindle assembly checkpoint kinase TTK enhances the efficacy of docetaxel in a triple-negative breast cancer model. Ann Oncol 2015;26:2180-92.

45. Zhu D, Xu S, Deyanat-Yazdi G, et al. Synthetic Lethal Strategy Identifies a Potent and Selective TTK and CLK1/2 Inhibitor for Treatment of Triple-Negative Breast Cancer with a Compromised G(1)-S Checkpoint. Mol Cancer Ther 2018;17:1727-38.

46. Szymiczek A, Carbone M, Pastorino S, et al. Inhibition of the spindle assembly checkpoint kinase Mps-1 as a novel therapeutic strategy in malignant mesothelioma. Oncogene 2017;36:6501-7.

47. Gordeeva O. Cancer-testis antigens: Unique cancer stem cell biomarkers and targets for cancer therapy. Semin Cancer Biol 2018;53:75-89.

48. Suda T, Tsunoda T, Daigo Y, et al. Identification of human leukocyte antigen-A24-restricted epitope peptides derived from gene products upregulated in lung and esophageal cancers as novel targets for immunotherapy. Cancer Sci 2007;98:1803-8.

49. Iinuma $H$, Fukushima R, Inaba T, et al. Phase I clinical study of multiple epitope peptide vaccine combined with chemoradiation therapy in esophageal cancer patients. J Transl Med 2014;12:84.

50. Kono K, Iinuma H, Akutsu Y, et al. Multicenter, phase II clinical trial of cancer vaccination for advanced esophageal cancer with three peptides derived from novel cancer-testis antigens. J Transl Med 2012;10:141.

51. Dong Z, Zhang $\mathrm{H}$, Zhan $\mathrm{T}$, et al. Integrated analysis of differentially expressed genes in esophageal squamous cell carcinoma using bioinformatics. Neoplasma 2018;65:523-31.

(English Language Editors: R. Scott and J. Reynolds)

Cite this article as: Yang Y, Sun Z, Shi Y, Sun J, Zhang X. Integrated bioinformatics analysis to identify key genes related to the prognosis of esophageal squamous cell carcinoma. Transl Cancer Res 2021;10(4):1679-1691. doi: 10.21037/tcr-20-3220 


\section{Supplementary}

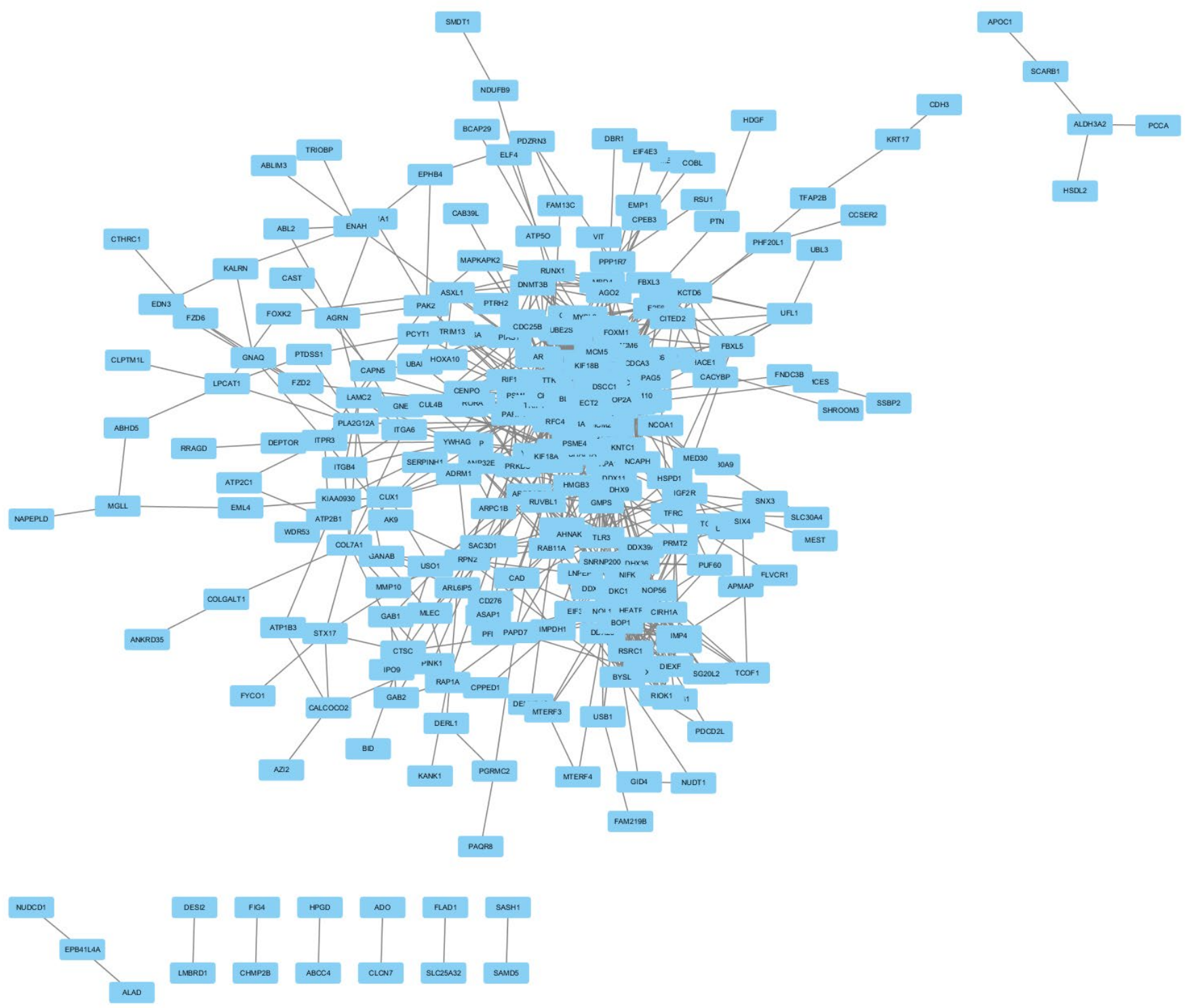

Figure S1 All differentially expressed genes in module analysis via Cytoscape. Each blue square represents a gene, and the connected lines represent interactions between genes. 

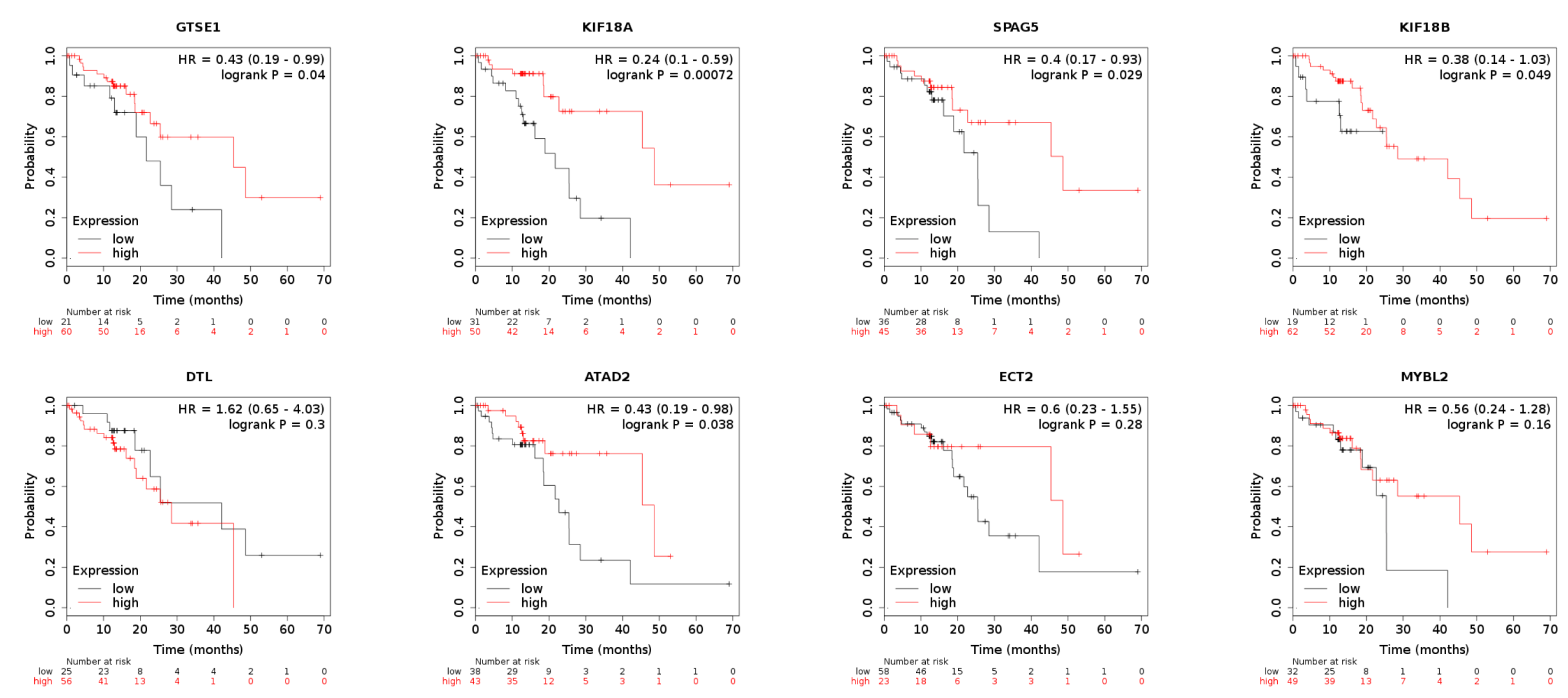

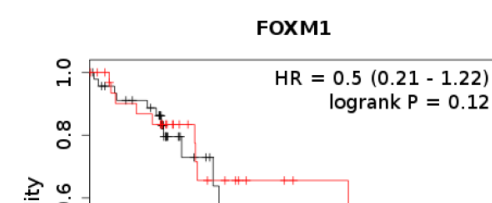

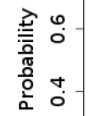

: Expression

$\begin{array}{llllllll}0 & 10 & 20 & 30 & 40 & 50 & 60 & \end{array}$

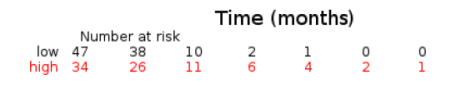

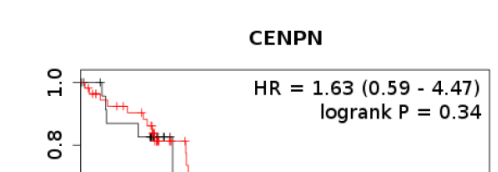

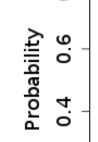

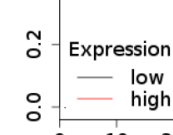

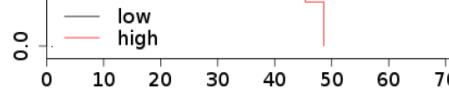

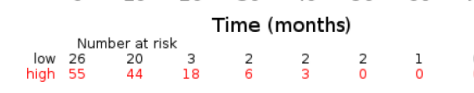

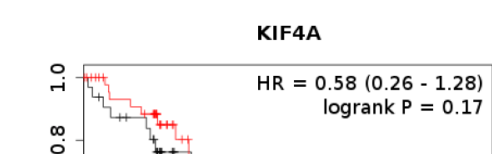

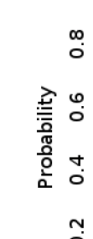

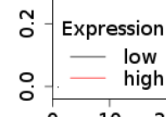

$\begin{array}{lllllll}10 & 20 & 30 & 40 & 50 & 60 & \end{array}$

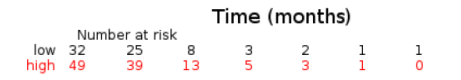

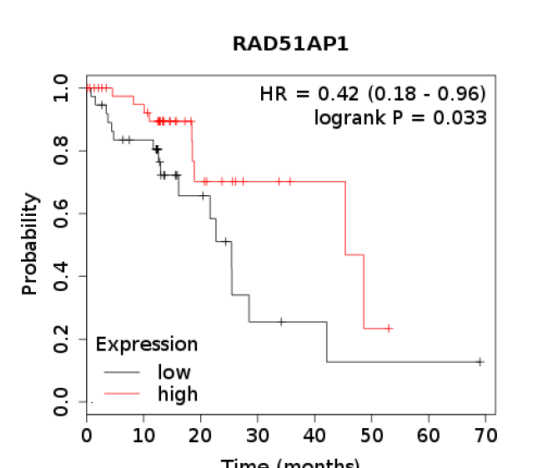

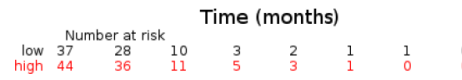

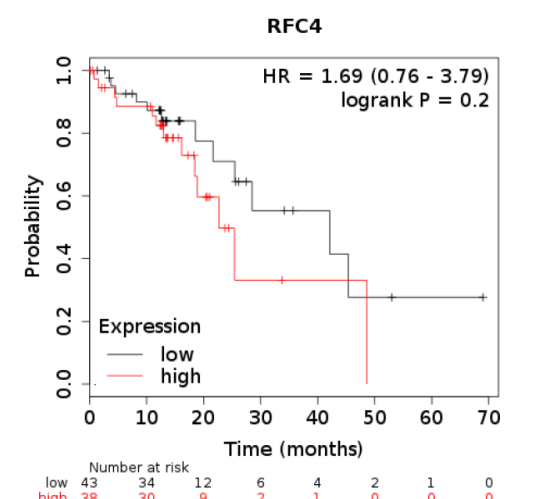

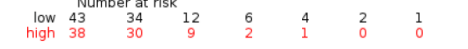
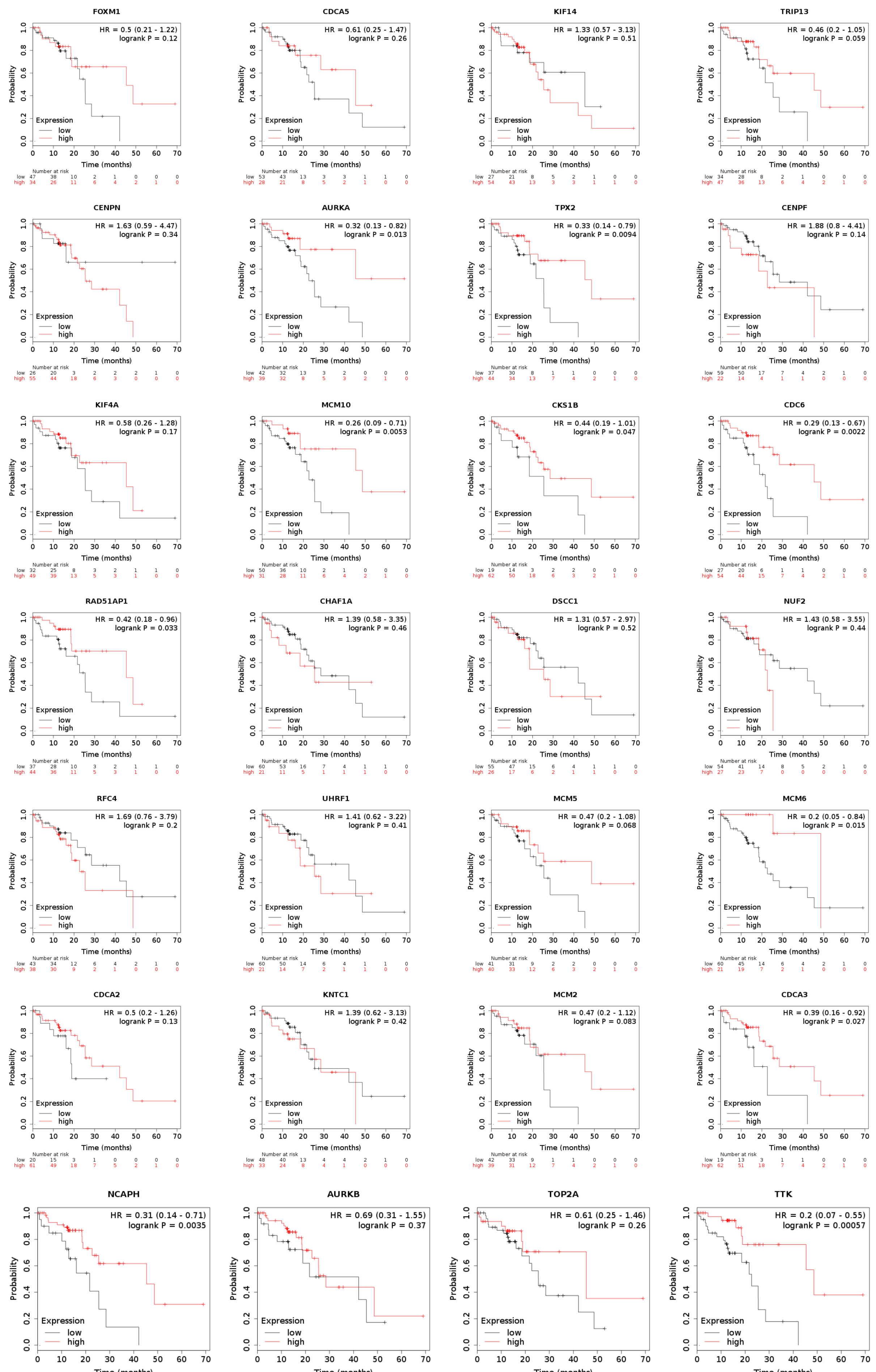

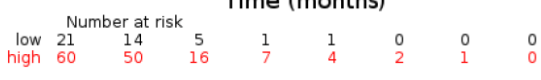
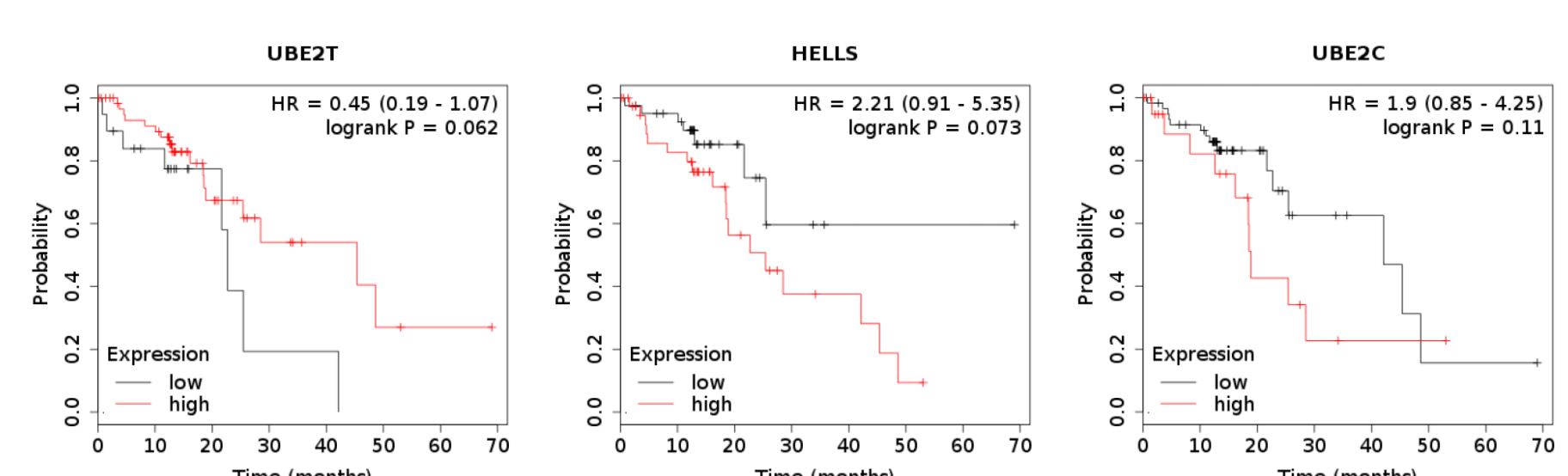

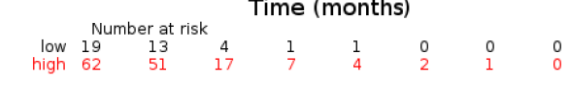

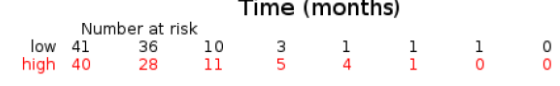

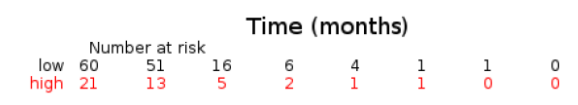

Figure S2 Survival curves of 39 hub genes (PICALM interacting mitotic regulator was excluded due to a lack of gene expression and survival information). Gene name is shown at the top of each figure part. Black line represents low expression of the identified gene, and red line represents high expression of the identified gene. Corresponding life table, hazard ratio, and log-rank P value are shown in each figure part. 